\title{
Impact of Preoperative Specialty Consults on Hospitalist Comanagement of Hip Fracture Patients
}

\author{
Nicholas Bellas, BS1, Sherry Stohler, RN, MSN², Ilene Staff, PhD³, Karolina Majk, MD², \\ Courtland Lewis, MD², Stephen Davis, MD², Mandeep Kumar, MD²
}

${ }^{1}$ University of Connecticut School of Medicine, Farmington, Connecticut; ${ }^{2}$ Hartford Healthcare Bone and Joint Institute, Hartford Hospital, Hartford, Connecticut; ${ }^{3}$ Department of Research Administration, Hartford Hospital, Hartford, Connecticut.

BACKGROUND: Hip fractures typically occur in frail elderly patients. Preoperative specialty consults, in addition to hospitalist comanagement, are often requested for preoperative risk assessment.

OBJECTIVE: Determine if preoperative specialty consults meaningfully influence management and outcomes in hip fracture patients, while being comanaged by hospitalists

DESIGN: Retrospective cohort study

SETTING: Tertiary care hospital in Connecticut

PATIENTS: 491 patients aged 50 years and older who underwent surgery for an isolated fragility hip fracture, defined as one occurring from a fall of a height of standing or less.

INTERVENTION: Presence or absence of a preoperative specialty consult

MEASUREMENTS: Time to surgery (TTS), length of hospital stay (LOS), and postoperative complications
RESULTS: 177 patients had a preoperative specialty consult. Patients with consults were older and had more comorbidities. Most consult recommendations were minor (72.8\%); there was a major recommendation only for eight patients (4.5\%). Multivariate analysis demonstrates that consults are more likely to be associated with a TTS beyond 24 hours (Odds Ratio [OR] 4.28 [2.79-6.56]) and 48 hours (OR 2.59 [1.52-4.43]), an extended LOS (OR 2.67 [1.78-4.03]), and a higher 30day readmission rate (OR 2.11 [1.09-4.08]). A similar 30-day mortality rate was noted in both consult and no-consult groups.

CONCLUSIONS: The majority of preoperative specialty consults did not meaningfully influence management and may have potentially increased morbidity by delaying surgery. Our data suggest that unless a hip fracture patient is unstable and likely to require active management by a consultant, such consults offer limited benefit when weighed against the negative impact of surgical delay. Journal of Hospital Medicine 2020;15:16-21. ( 2020 Society of Hospital Medicine for ip fractures in the elderly are associated with significant morbidity and mortality. ${ }^{1}$ These are typically fragility fractures since they are caused by mechanical forces that would ordinarily not result in a serious injury, such as a fall from or below standing level. The incidence of hip fractures in the United States is expected to increase as the population ages; estimates project 512,000 hip fractures with an associated cost of $\$ 16$ billion annually by the year $2040 .^{2}$ Timely surgery is recommended for hip fracture patients as delayed surgery beyond 24 to 48 hours of presentation is associated with increased morbidity and mortality. ${ }^{3-6}$ Time to surgery (TTS) has been shown to be the major potentially modifiable risk factor in the management of a hip fracture.

Factors that have been noted to influence TTS include the American Society of Anesthesiologists' (ASA) score, the day of the week of hospital admission, and preoperative testing. . $^{89}$ Preoperative cardiology consultation and subsequent cardiac testing, in

*Corresponding Author: Mandeep Kumar, MD; E-mail: mandeep88@gmail. com; Telephone: 860-972-0549

Published online first August 21, 2019.

Received: January 8, 2019; Revised: June 5, 2019; Accepted: June 5, 2019

(c) 2020 Society of Hospital Medicine DOI 10.12788/jhm.3264 particular, can increase the TTS and length of stay (LOS) without changing perioperative management. ${ }^{9,10}$ In our review of literature, we could not identify any studies specifically looking at the impact of preoperative specialty consults on short-term mortality or comparison of care provided by hospitalists alone versus additionally involving subspecialists such as cardiologists. To our knowledge, there are no studies that have categorized recommendations from a preoperative specialty consult as minor, moderate, or major.

Our study evaluated whether preoperative specialty consults meaningfully change management and influence outcomes for hip fracture patients. At our institution, all hip fracture patients are admitted to the hospitalist service and comanaged with the orthopedic team. The hospitalist physician performs the preoperative evaluation as part of the admission history and physical exam. Preoperative specialty consult(s), if needed, are requested only by the hospitalist team. A consultant such as a cardiologist provides input; however, final management decisions are coordinated by the hospitalist physician.

\section{METHODS}

\section{Study Design}

We performed a retrospective cohort study of patients aged 50 years and older who underwent surgery for an isolated fragility fracture of the hip at Hartford Hospital, a level one trauma 
and tertiary care medical center, within the 24-month period from April 2015 to March 2017. Fragility hip fracture is defined as one occurring from a fall of a height of standing or less. A consult referred to a specialty or subspecialty consultation, other than hospital medicine, obtained prior to surgery. $\mathrm{Pa}$ tients with additional skeletal trauma and periprosthetic fractures were excluded. A total of 491 unique patients met the inclusion criteria, and data were obtained from chart review and an orthopedic surgery registry. The Hartford Hospital Institutional Review Board approved this study.

Our primary predictor was the presence or absence of a preoperative specialty consultation requested by the hospitalist. We also analyzed the following: covariates of demographics (age, sex, race), the ASA score, and severity of comorbidities using the Charlson comorbidity index (CCl) with a Quan modification;" "R program package, International Classification of Disease (ICD)"12 was used to calculate the CCl using ICD-9 and ICD-10 diagnostic codes.

The primary outcome measures were TTS (measured in hours), LOS (measured in days), complications, and preoperative specialty consult resulting in a change in perioperative management. TTS was defined as the time elapsed from the presentation at the emergency department (ED) to surgery start. For transfer or direct admission patients, the time of admission was used in place of time of presentation. The measured complications included postoperative venous thromboembolic events, surgical site infection, myocardial infarction, stroke, and sepsis. Secondary outcome measures included 30day mortality, readmission rate, and rate of return to OR. There were no elective or planned readmissions postoperatively on review of our institution's orthopedic surgery registry.

Our team performed an extensive chart review including reviewing the admission note, consulting physician notes, and relevant test results. Our senior investigator (MK) then rated each preoperative specialty consult on appropriateness, the relative strength of the consultant's recommendation, and resulting change in perioperative management. Cardiology consultations were deemed reasonable if a patient's cardiac risk was considered elevated by the admitting physician or an active cardiac condition was present (suggestion of or clear evidence for acute coronary syndrome, acute congestive heart failure, uncontrolled arrhythmia, or symptomatic valvular disease). The determination of "elevated cardiac risk" was made, if admit note contained verbiage expressing concern for further evaluation for cardiac issues or words such as "high risk" or "elevated risk". A specific guideline-based score such as the revised cardiac risk index was not consistently available in this retrospective chart review. A noncardiology consult was deemed reasonable only if it would have been warranted for the specific clinical situation-for example, a neurology consult for an acute stroke or a pulmonary consult for acute respiratory failure. Consult recommendations or outcomes were rated as minor, moderate, or major (see Table 1 for detailed criteria). Some consults may generate more than one recommendation, in these cases, we determined that a major recommendation supersedes a moderate or minor recommendation and only one was counted in the final analysis. Next we determined if a consult recommendation led to a change in perioperative or therapeutic management, defined as a medication or dosage change, need to delay surgery to stabilize an unstable medical condition, invasive procedures (such as thoracentesis or cardiac catheterization) or change in postoperative monitoring. As a way of clarification, a consult may have a minor recommendation such as an EKG but if no other recommendations were given and there was no change in therapeutic management such as a medication change, this would be considered as a "no change".

An independent rating of the entire dataset was subsequently performed by another hospitalist (KM) to establish interrater reliability. This reviewer was blinded to the initial rating and not involved in the initial design of the study or the data collection process. Because of the labor-intensive task of reviewing full charts, we followed a nonstandard process for interrater reliability. This rating was performed with the same dataset that was extracted by three members of our team (NB, SS, and MK); consequently, this does not account for variability in chart extraction as reiterated in the discussion.

\section{Statistical Analysis}

The main analyses compared the two patient subgroups (with or without preoperative specialty consults) around outcome measures. Primary outcome measures were TTS, LOS, complications, and consult resulting in a change in perioperative management. Secondary outcome measures were 30-day readmit, return to $\mathrm{OR}$, and mortality. A preliminary analysis was conducted to explore distributions for TTS and LOS. As expected, none met the assumptions of normality and were thus analyzed with Wilcoxon ranked-sum tests. The other outcomes were dichotomous and analyzed with chi-square tests of proportion or Fisher's exact test when the expected cell frequencies were too low. Dichotomized variables for TTS (within 24 hours and 48 hours) and LOS (within five days, the median LOS for this cohort) were calculated and subsequently analyzed with additional chi-square tests of proportion or Fisher's exact test ${ }^{13}$. To explore the effect of preoperative specialty consults independent of potential confounders, logistic regression analyses predicting each of the dichotomous outcomes were conducted with age and $\mathrm{CCl}$ used as predictors in addition to the main variable of whether or not there was a preoperative specialty consult. Since the $\mathrm{CCl}$ and ASA scores were highly intercorrelated, only the former was chosen for the multivariate analyses based on the consistent algorithm used to calculate $\mathrm{CCl}$.

Additional analyses with the subgroup of patients with a preoperative specialty consult explored whether the consult was reasonable, the relative strength of resulting recommendation and whether it resulted in a change in management. The statistical approach used was the same as for the other dichotomous outcomes. All analyses used 0.05 as the level of statistical significance; SPSSv21 (IBM, Armonk, New York) was the statistical software used.

The sample size for this retrospective analysis was determined by the available number of patients meeting the inclusion criteria. An a priori power calculation was done to 
TABLE 1. Consult Outcome Classification

Classification of Minor, Moderate, and Major Recommendation of Consultation (number of patients)

\begin{tabular}{|c|c|c|}
\hline Minor (129) & Moderate (40) & Major (8) \\
\hline $\begin{array}{l}\text { Ordering an Electrocardiogram or pacemaker evaluation or } \\
\text { interpret existing information/studies (79) }\end{array}$ & Perform Echocardiogram or stress test preoperatively (29a) & $\begin{array}{l}\text { Preoperative urgent or emergent surgery (coronary artery bypass } \\
\text { graft, pericardial window, etc.; } 1 \text { ) }\end{array}$ \\
\hline $\begin{array}{l}\text { Initiation of new medication or change of dose or timing of a } \\
\text { current medication ( } 42 \text { ) }\end{array}$ & Anticipated postoperative intensive care or step-down unit care (2) & $\begin{array}{l}\text { Invasive diagnostic test such as a cardiac catheterization } \\
\text { (one patient had aortic valvuloplasty performed concurrently) }\end{array}$ \\
\hline $\begin{array}{l}\text { Evaluation of anticoagulation status-delaying surgery due to } \\
\text { active anticoagulant (8) }\end{array}$ & $\begin{array}{l}\text { Stabilization of existing conditions such as congestive heart failure } \\
\text { or dysrhythmias (9) }\end{array}$ & $\begin{array}{l}\text { Interventional procedure such as thoracentesis, valvuloplasty, } \\
\text { IVC filter placement, or cholecystostomy placement (6) }\end{array}$ \\
\hline
\end{tabular}

Abbreviation: IVC, inferior vena cava.

TABLE 2. Patient Demographics of the Consult and No-Consult Groups $(n=491)^{\text {a }}$

\begin{tabular}{|c|c|c|c|}
\hline & No Consult (314) & Consult (177) & $P$ Value \\
\hline Female gender-n (\%) & $227(72.3)$ & $121(68.4)$ & .357 \\
\hline Race-n (\%) & & & .884 \\
\hline Caucasian & $274(89.8)$ & $156(88.6)$ & \\
\hline African American & $12(3.9)$ & $7(4.0)$ & \\
\hline Latino & $12(3.9)$ & $7(4.0)$ & \\
\hline Other (including Asian) & $7(2.3)$ & $6(3.4)$ & \\
\hline Greater than 80 years old-n (\%) & $193(61.5)$ & $134(75.7)$ & $<.001$ \\
\hline Age median, (IQR) & $83(74,89)$ & $86(80,91)$ & $<.001$ \\
\hline BMI median, (IQR) & $24(21,28)$ & $23(21,27)$ & .186 \\
\hline Charles comorbidity index (median, (IQR) & $1(0,3)$ & $3(2,4)$ & $<.001$ \\
\hline ASA score (median, IQR) & $3(2,3)$ & $3(3,4)$ & $<.001$ \\
\hline Type of anesthesia-n (\%) general vs spinal & $218(69.4)$ & $122(68.9)$ & .908 \\
\hline Day of the week $n(\%)$ on weekend & $107(34.1)$ & $51(28.8)$ & .231 \\
\hline
\end{tabular}

${ }^{a}$ Statistically significant numbers are bolded.

Abbreviations: ASA, American Society of Anesthesiologists; IQR, interquartile range.

determine if the expected volume would be sufficient for the multivariate analysis; the presence of a complication was selected for calculation. Based on an expected volume of approximately 500 and an estimate of a $10 \%$ serious complication rate, it was determined that the sample could support the analysis of up to five predictor variables, sufficient for the main variable and four potential confounders; this was considered adequate. ${ }^{14}$ Propensity scoring was considered but did not offer any advantages to logistic regression because we only had two observed covariates: $\mathrm{CCl}$ and age.

\section{RESULTS}

A total of 491 unique patients met our inclusion criteria, 177 patients had a preoperative specialty consult. Of these $177 \mathrm{pa}-$ tients, 24 patients had more than one consult; hence, the total number of consults was 201. Most of the consults were cardiology (159). Others were Infectious disease (11), Pulmonology (10), Neurology (7), and Miscellaneous (14, which included Nephrology, Gastroenterology, Hematology, and Oncology).

No significant differences were found between the consult and no-consult groups with respect to gender, race, body mass index, type of anesthesia, and day of the week of surgery. We did note that patients with a consult were older and had a significantly higher $\mathrm{CCl}$ and ASA score (Table 2).

Initial analyses compared those with and without consults unadjusted for other factors with respect to TTS, LOS, 30-day readmission rate, 30-day return to OR rate, and 30-day mortality rate. The median TTS was 22.1 hours for the no-consult group compared with 34.3 hours for the consult group. The percentage of patients with TTS within 24 hours was higher 
TABLE 3. Unadjusted Outcome Measures for the Consult and No Consult Groups $(\mathrm{N}=491)^{\mathrm{a}}$

\begin{tabular}{|c|c|c|c|}
\hline & No consult (314) & Consult (177) & $P$ Value \\
\hline Time to surgery (in hours) - median (IQR) & $22.1(17.1,29.4)$ & $34.3(24.4,47.1)$ & $<.001$ \\
\hline Length of Stay (in days)- median (IQR) & $5(4,6)$ & $6(5,8)$ & $<.001$ \\
\hline TTS within 24 hours - $\mathrm{N}(\%)$ within time period & $184(58.6)$ & $42(23.7)$ & $<.001$ \\
\hline TTS within 48 hours $-\mathrm{N}(\%)$ within time period & $283(90.1)$ & $136(76.8)$ & $<.001$ \\
\hline Serious complication - N (\%) & $16(5.1)$ & $11(6.2)$ & .601 \\
\hline Any complication - N (\%) & $167(53.2)$ & $108(61.0)$ & .093 \\
\hline Intraoperative complication- N (\%) & $2(0.6)$ & $1(0.6)$ & 1.00 \\
\hline 30-day readmission $\mathrm{N}(\%)$ & $21(6.7)$ & $23(13.0)$ & .02 \\
\hline 30-day return to Operating room - N (\%) & $1(0.3)$ & $3(1.7)$ & .137 \\
\hline 30-day mortality- $\mathrm{N}(\%)$ & $9(2.9)$ & $16(9.0)$ & .003 \\
\hline
\end{tabular}

a Statistically significant numbers are bolded.

Abbreviations: IQR, Interquartile Range; TTS, time to surgery.

TABLE 4. Relationship Between Consults and Outcome Measures (Unadjusted and Adjusted)

\begin{tabular}{|c|c|c|c|c|c|}
\hline Outcome & \multicolumn{5}{|c|}{ CONSULTS } \\
\hline TTS beyond 24 hours $^{\mathrm{a}}$ & 4.55 & $3.01-6.87$ & 4.28 & $2.79-6.56$ & $<.001$ \\
\hline TTS beyond 48 hours $^{\mathrm{a}}$ & 2.75 & $1.65-4.58$ & 2.59 & $1.52-4.43$ & $<.001$ \\
\hline 30-day readmission rate & 2.07 & $1.11-3.86$ & 2.11 & $1.09-4.08$ & .027 \\
\hline 30-day mortality rate & 3.37 & $1.46-7.79$ & 2.2 & $0.92-5.25$ & .076 \\
\hline Serious complication rate & 1.23 & $0.56-2.72$ & 0.94 & $0.41-2.13$ & .875 \\
\hline
\end{tabular}

aindicates having one or more consults is a statistically significant, independent predictor of listed outcome.

${ }^{b}$ All statistically significant odds ratios are bolded.

Abbreviations: LOS; length of stay; OR, odds ratio; TTS, time to surgery.

(58.6\% compared with 23.7\%) and TTS within 48 hours was higher (90.1\% compared to $76.8 \%$ ) if there was no consult. The median LOS was five days for the no-consult group compared with six days for the consult group. There was no difference in complications between the two groups. Patients with consults were more likely to have a readmission (Table 3). No association was found between the type of consult (cardiology, pulmonary, etc.) and outcomes.

In the main analyses adjusted for potential confounders of age and $\mathrm{CCl}$, consults were more likely to be independently associated with TTS beyond 24 hours, TTS beyond 48 hours, an extended LOS, and a higher 30-day readmission rate. $\mathrm{CCl}$ independently predicted a higher LOS, 30-day mortality rate, and serious complication rate. Similarly, age predicted 30-day mortality. Consults were not independently associated with 30day mortality (Table 4).

Of the 177 patients with one or more consults, 163 (92\%) were deemed reasonable. Of the patients, 129 (72.8\%) had minor, 40 (22.6\%) moderate, and 8 (4.5\%) major recommendations as a result of the consultation. There was an identifiable change in perioperative management for 66 (37\%) patients with consults. The independent review done for interrater reliability examined the entire dataset. This review demonstrated the following percent agreements: $99.4 \%$ for if the consult was indicated (kappa $=0.962$ ), 97.7\% for the consult outcome classification (minor, moderate, or major; kappa $=0.947$ ), and $94.4 \%$ for if the intervention resulted in a change in management (kappa $=0.878)$. 
While reviewing our subset of cardiology consults, we noted moderate or major recommendations from a cardiologist only in cases where an active cardiac condition was suspected by the hospitalist requesting the consult. Only eight patients in our study had major recommendations from a consult, of which, three underwent aortic valvuloplasty and one patient each underwent the following: pericardial window for tamponade, cholecystostomy tube placement to treat acute cholecystitis, thoracentesis, endoscopic retrograde cholangiopancreatography for obstructive jaundice, and inferior vena cava filter placement for acute pulmonary embolism. All these procedures were done prior to hip fracture repair. Interestingly, 42 out of the 177 patients in our consult group had a preoperative echocardiogram performed, with only three patients with critical aortic stenosis undergoing valvuloplasty preoperatively.

\section{DISCUSSION}

Patients with preoperative specialty consults were older and had more comorbidities than patients without consults. Our findings suggest that consults contribute to delays to surgery and may lead to higher LOS and higher risk of 30-day readmission after controlling for age and comorbidities in a multivariate analysis. This observation is significant considering that consults were requested more frequently on patients with a higher comorbidity burden and included patients who did not get additional preoperative testing, suggesting that a delay from waiting for a consult alone may be deleterious. This was a unique observation in our study; prior studies examining this subject have attributed delays to additional testing and not consults alone. Even though most consult requests appear to be reasonable according to our criteria, the majority of recommendations were minor $(72.9 \%)$, and $62.7 \%$ of consults resulted in no change in perioperative management. Major changes in perioperative management were noted in only $4.5 \%$ of patients.

Our finding that a majority of patients in the consult group had no significant change in perioperative management raises an important area of potential improvement in the care of hip fracture patients. We believe that narrowing indications for preoperative specialty consults may result in shorter TTS and LOS for this group of frail elderly patients without sacrificing the quality of care. Since all patients in our study were comanaged by hospitalists and patients without additional consults had similar or better outcomes, we believe that hospitalist physicians are well positioned to provide standardized comanagement to this patient group without additional consultation unless absolutely necessary.

The primary limitation of our study was that this was a retrospective case analysis. The designation of minor, moderate, or major recommendation was done after the consults were already completed, and it may not be possible to predict that a consult results in no change without it being actually performed. Additionally, our classification of recommendations is somewhat arbitrary and subjective; for example, some readers might argue that a medication change counts as a moderate recommendation. We rated a medication change to be minor as we believe that an experienced hospitalist may likely make such management decisions on their own, and if this is the only recommendation from a consult, it is not additional information critical to patient care. There may also be an "unmeasured complexity" noted by the admitting physician, which was not necessarily accounted for by multivariate analysis of age and $\mathrm{CCl}$ but one that led to higher mortality and readmissions. However, we feel that this "unmeasured complexity" is likely inconsequential as the vast majority of consults did not result in any change in management. We did adjust for covariates as noted, but some confounding by indication is likely to remain. Additionally, categorization of consult recommendations and consequent changes by one physician could be considered subjective. We did control for this by having another physician review the entire dataset and rate it independently for interrater reliability with excellent correlation and kappa, although these may be inflated to some degree because our chart review did not account for variability among chart extractors.

A prospective evaluation of a clinical protocol that delineates reasonable indications for a preoperative consult would be helpful to validate our findings. In our study, we noted moderate or major recommendations from a cardiologist only in cases where an active cardiac condition was suspected by the hospitalist requesting the consult; hence, limiting preoperative cardiology consults to active cardiac conditions may be a reasonable approach to evaluate in a prospective study.

In conclusion, a majority of preoperative specialty consults do not appear to meaningfully influence management and may indirectly increase morbidity by delaying surgery and extending hospital stays. Our data suggest that unless the patient is clinically unstable and likely to require active management by a consultant prior to hip fracture repair, consults may offer limited benefit. Appropriately standardized perioperative management of this patient group by hospitalist physicians appears to manage most hip fracture patients as effectively with faster TTS and shorter hospital LOS.

\section{Acknowledgments}

The authors would like to thank John Corradi, PhD (Research Department at Hartford Hospital) for his input in calculating the Charlson comorbidity index. ${ }^{12,13}$

Disclosures: The ICMJE COI form was reviewed by all authors and they have nothing to disclose.

\section{References}

1. Youm T, Koval KJ, Zuckerman JD. The economic impact of geriatric hip fractures. Am J Orthop. 1999;28(7):423-428.

2. Cummings $S R$, Rubin $S M$, Black D. The future of hip fractures in the United States: numbers, costs, and potential effects of post-menopausal estrogen. Clin Orthop Relat Res. 1990;(252):163-166

3. Mitchell SM, Chung AS, Walker JB, Hustedt JW, Russell GV, Jones CB. Delay in hip fracture surgery prolongs postoperative hospital length of stay but does not adversely affect outcomes at 30 days. J Orthop Trauma. 2018;32(12):629-633. https://doi.org/10.1097/BOT.0000000000001306.

4. Sobolev B, Guy P, Sheehan KJ, et al. Mortality effects of timing alternatives for hip fracture surgery. CMAJ. 2018;190(31):E923-E932. https://doi.org/10.1503/ cmaj.171512.

5. Pincus D, Ravi B, Wasserstein D, et al. Association between wait time 
and 30-day mortality in adults undergoing hip fracture surgery. JAMA 2017;318(20):1994-2003. https://doi.org/10.1001/jama.2017.17606.

6. Fu MC, Boddapati V, Gausden EB, Samuel AM, Russell LA, Lane JM. Surgery for a fracture of the hip within 24 hours of admission is independently associated with reduced short-term post-operative complications. Bone Joint J. 2017;99B(9):1216-1222. https://doi.org/10.1302/0301-620X.99B9.BJJ-2017-0101.R1.

7. Belmont PJ Jr, Garcia EJ, Romano D, Bader JO, Nelso KJ, Schoenfeld AJ. Risk factors for complications and in-hospital mortality following hip fractures: a study using the National Trauma Data Bank. Arch Orthop Trauma Surg. 2014;134(5):597-604. https://doi.org/10.1007/s00402-014-1959-y.

8. Ricci WM, Brandt A, McAndrew C, Gardner MJ. Factors affecting delay to surgery and length of stay for patients with hip fracture. J Orthop Trauma. 2015;29(3):e109-e114. https://doi.org/10.1097/BOT.0000000000000221.

9. Bernstein J, Roberts FO, Wiesel BB, Ahn J. Preoperative testing for hip fracture patients delays surgery, prolongs hospital stays, and rarely dictates care. J Orthop Trauma. 2016;30(2):78-80. https://doi.org/10.1097/ BOT.0000000000000444

10. Ricci WM, Della Rocca GJ, Combs C, Borrelli J. The medical and economic impact of preoperative cardiac testing in elderly patients with hip fractures. Injury. 2007;38(supp/ 3):S49-S52. https://doi.org/10.1016/j.injury.2007.08.011.

11. Quan $\mathrm{H}$, Li B, Couris CM, et al. Updating and validating the Charlson comorbidity index and score for risk adjustment in hospital discharge abstracts using data from 6 countries. Am J Epidemiol. 2011;173(6):676-682. https:// doi.org/10.1093/aje/kwq433

12. Wasey JO. icd: Tools for working with ICD-9 and ICD-10 codes, and finding comorbidities. R package version 3.2.0. https://CRAN.R-project.org/package=icd. Published 2018. Accessed November 13, 2018.

13. Uitenbroek D. The Fisher exact test for $2 * 5$ or smaller crosstable. Quantitativeskills.com. https://www.quantitativeskills.com/sisa/statistics/fiveby2.htm. Published 2019. Accessed November 13, 2018

14. Peduzzi P, Concato J, Kemper E, Holford TR, Feinstein AR. A simulation study of the number of events per variable in logistic regression analysis. J Clin Epidemiol. 1996;49(12):1373-1379. https://doi.org/10.1016/S08954356(96)00236-3.

\section{ure-Na \\ for hyponatremia}

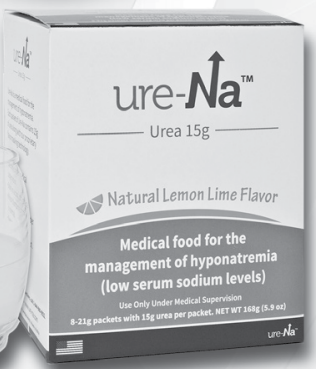

$\checkmark$ Guideline supported $\checkmark$ Safe and cost effective

ure-Na may already be available on your in-patient formulary. If it's not, ask pharmacy to review it for formulary inclusion.

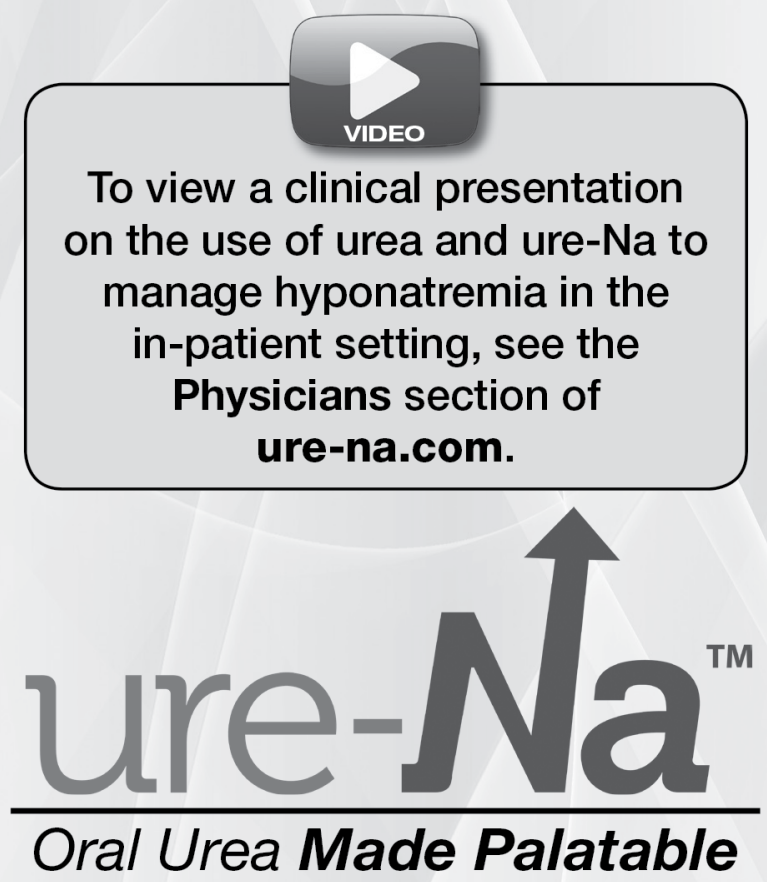

*The European Clinical Practice Guideline on the management of hyponatremia recommend the use of oral urea as a treatment option in SIADH for moderate to profound hyponatremia. UpToDate also reviews the use of urea as a management option for hyponatremia.

\section{Learn more at} ure-na.com 